\title{
Zooming towards the Event Horizon - mm-VLBI today and tomorrow
}

Thomas P. Krichbaum*, A. Roy, J. Wagner, H. Rottmann, J. A. Hodgson, A. Bertarini, W. Alef, J. A. Zensus

Max-Planck-Institut für Radioastronomie, 53121 Bonn, Germany

E-mail: tkrichbaumempifr.de

\section{A.P. Marscher, S.G. Jorstad}

Institute for Astrophysical Research, Boston University, Boston, MA 02215, USA

R. Freund, D. Marrone, P. Strittmatter, L. Ziurys

Arizona Radio Observatory, Tucson, AZ 85721, USA

R. Blundell, J. Weintroub, K. Young

Harvard Smithsonian Center for Astrophysics, Cambridge, MA 02138, USA

V. Fish, S. Doeleman

MIT Haystack Observatory, Westford, MA 01886, USA

M. Bremer, S. Sanchez

Institut de Radioastronomie Millimétrique (IRAM), 38406 St Martin d'Hères, France

\section{Fuhrmann, E. Angelakis, V. Karamanavis}

Max-Planck-Institut für Radioastronomie, 53121 Bonn, Germany

Global VLBI imaging at millimeter and sub-millimeter wavelengths overcomes the opacity barrier of synchrotron self-absorption in Active Galactic Nuclei (AGN) and opens the direct view into sub-pc scale regions not accessible before. Since AGN variability is more pronounced at short millimeter wavelength, mm-VLBI can reveal structural changes in very early stages after outbursts. When combined with observations at longer wavelength, global $3 \mathrm{~mm}$ and $1 \mathrm{~mm}$ VLBI adds very detailed information on the source structure. This helps to determine fundamental physical properties at the jet base, and in the vicinity of super-massive black holes at the center of AGN. Here we present new results from multi-frequency mm-VLBI imaging of OJ 287 during a major outburst. We also report on a successful $1.3 \mathrm{~mm}$ VLBI experiment with the APEX telescope in Chile. This observation sets a new world record in angular resolution. It also opens the path towards future mm-VLBI with ALMA, which aims at the mapping of the black hole event horizon in nearby galaxies, and the study of the roots of jets in AGN.

11th European VLBI Network Symposium \& Users Meeting,

October 9-12, 2012

Bordeaux, France

\footnotetext{
${ }^{*}$ Speaker.
} 


\section{Introduction}

In Active Galactic Nuclei (AGN) the energy extraction from black holes (BH) and the detailed understanding of jet formation and jet acceleration are still poorly understood. It is therefore desirable to observe galaxies with black holes and their emanating jets with an as high as possible angular and spatial resolution. For nearby super massive black holes, such as the BH in the Galactic Center (D $\sim 8 \mathrm{kpc}$ ) and in M 87 (Virgo $\mathrm{A}, \mathrm{D} \sim 17 \mathrm{Mpc}$ ) ground-based VLBI at $\lambda \leq 1.3 \mathrm{~mm}$ $(v \geq 230 \mathrm{GHz})$ provides an observing beam well matched to the expected size of the event horizon - and by this to the size of the observable emission region around such black holes. For the more distant quasars (and other AGN) a spatial resolution of several 10-1000 gravitational radii is obtained. Two fundamentally different types of jet formation models are often discussed: (i) in Blandford-Payne (BP) type models the particle acceleration is done via a magnetic sling-shot mechanism with field lines anchored in the rotating accretion disk collimating and accelerating a disk wind. (ii) In Blandford-Znajek (BZ) type models the energy extraction is purely electromagnetic and is directly coupled to the spin of the black hole. As a consequence of this, the diameter and morphology of the jet base (i.e. at radii where the jet emission becomes observable) may be different for BP- and BZ-type jet launching, in the sense that BZ-jets may appear more narrow and compact. VLBI imaging with ten micro-arcsecond scale resolution can help to discriminate between these models and constrain the parameter space for the theoretical modeling.

\section{Global VLBI at $3 \mathrm{~mm}$ wavelength}

Global $3 \mathrm{~mm}$ VLBI imaging at $86 \mathrm{GHz}$ is performed with the stand-alone VLBA (8 antennas equipped with $3 \mathrm{~mm}$ receivers, no receiver at $\mathrm{HN}$ and $\mathrm{SC}$ ) and with the Global Millimeter VLBI Array (GMVA). The GMVA combines the big European antennas (100 m Effelsberg, $30 \mathrm{~m}$ IRAM Pico Veleta, 6 x 15 m IRAM Plateau de Bure, 40 m Yebes, 20 m Onsala, 14 m Metsähovi) with the VLBA. While the VLBA could observe more frequently, the GMVA is about 3 times more sensitive and offers also a much higher resolution than the VLBA (up to $40 \mu$ as). A brief description of the present status of the GMVA and new results are presented by J. Hodgson et al., in this conference [5] (and references therein).

VLBI imaging with the highest possible angular (and spatial) resolution can shed light on the physical processes acting within the centers of blazars, in regions where jets are forming and gamma-rays are produced. As an example we show in Figure 1 three VLBI maps of the prominent blazar OJ 287 observed in October 2009 at 15 \& $43 \mathrm{GHz}$ with the VLBA, and at $86 \mathrm{GHz}$ with the GMVA. At the lower frequencies, OJ 287 shows a bent core-jet structure, with a prominent and unresolved core and some faint jet-emission extending west to $r \leq 5$ mas. At $86 \mathrm{GHz}$, however, the core regions breaks up into two bright sub-components ( $\mathrm{C} 1 \& \mathrm{C} 2)$, which interestingly are oriented along a line almost perpendicular to the direction of the mas-scale jet. If interpreted by a spatially bent jet, one would expect the northern most component (C1) to be the VLBI core, which is the unresolved base of a synchrotron self-absorbed jet. In Figure 2 we show the $43 \mathrm{GHz}$ and $86 \mathrm{GHz}$ maps convolved with a circular beam of 0.1 mas in size (factor $\sim 2$ super-resolution at $43 \mathrm{GHz}$ ). The two components seen at $86 \mathrm{GHz}$, now are also clearly visible at $43 \mathrm{GHz}$. In October 2009 , OJ 287 was in the rising phase of a prominent radio-flare (peak: Jan. 2010), which followed a strong gamma-ray flare (peak: October 24, 2009, see [2]). In Figure 3 we show the radio lightcurves from the F-GAMMA program [3], from which we determine two radio spectra, one near 

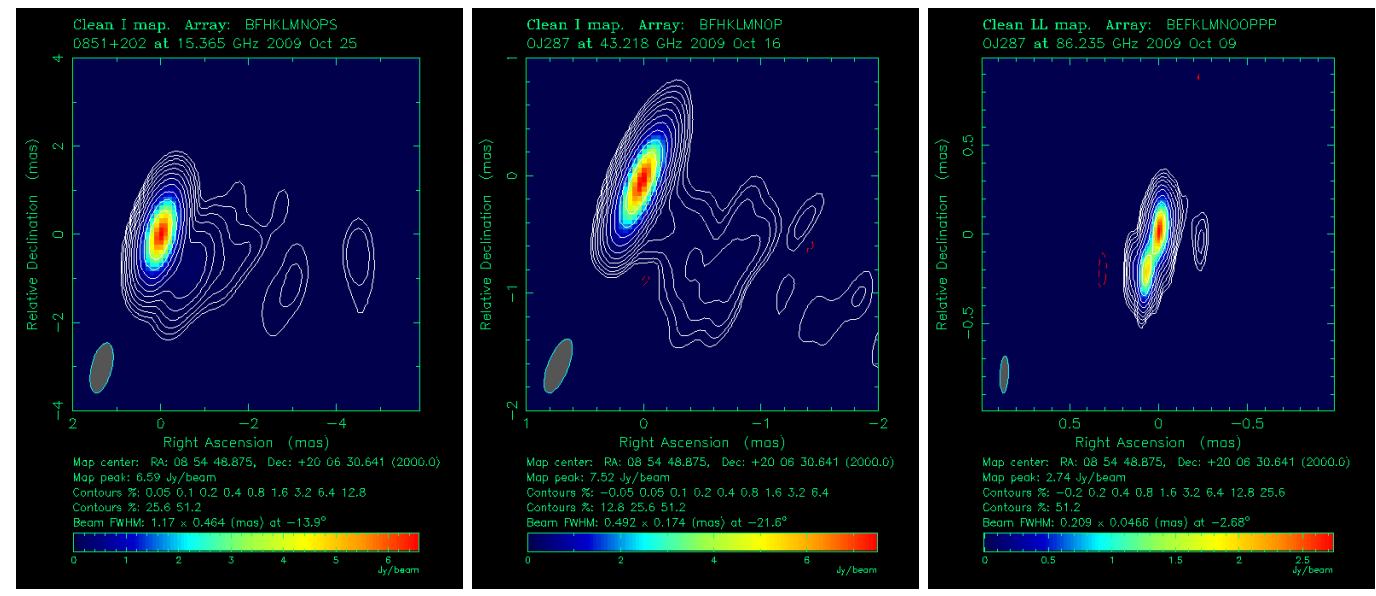

Figure 1: Quasi-contemporanous VLBI maps of OJ287 at $15 \mathrm{GHz}$ (left; data: Mojave data base), $43 \mathrm{GHz}$ (center, data: Boston group), and $86 \mathrm{GHz}$ (right, data: GMVA) of October 2009.
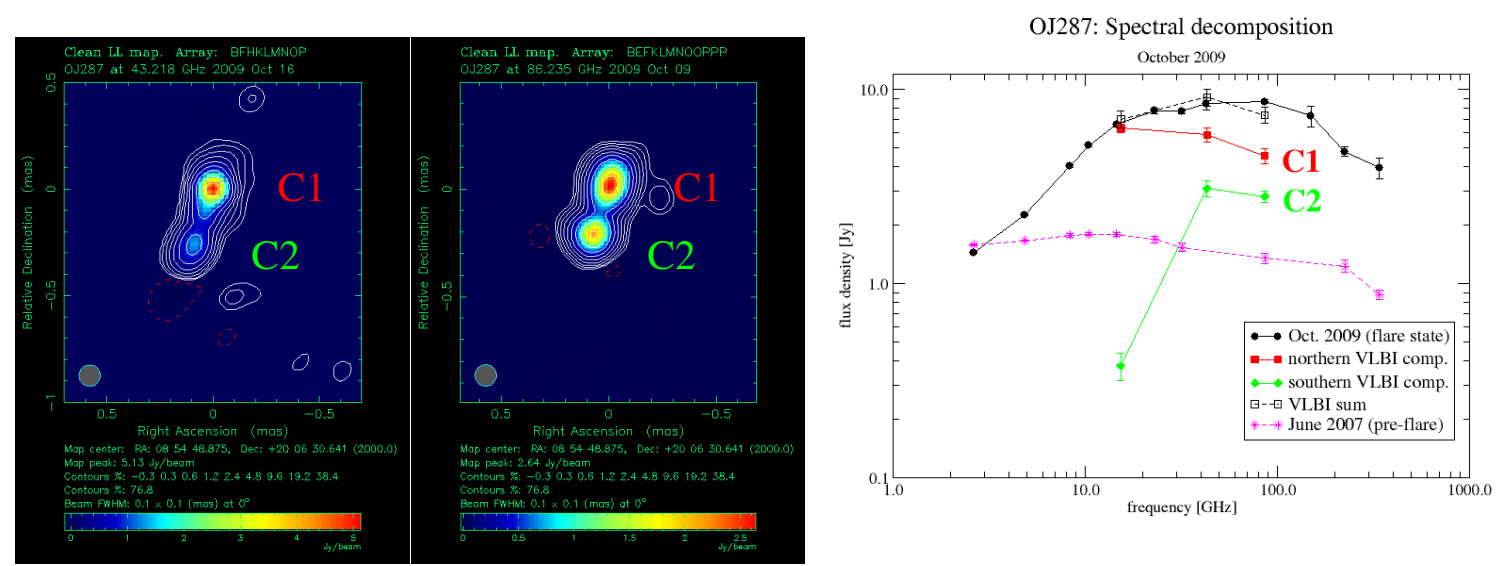

Figure 2: Left: $43 \mathrm{GHz}$ VLBI image of OJ287 from VLBA. Center: $86 \mathrm{GHz}$ VLBI image of OJ287 from GMVA. Both maps are convolved with a circular beam of 0.1 mas size for better comparison. Right: Spectral decomposition. Spectra of the VLBI components C1 and C2 and the total radio total spectrum (black: 2009 - flare state, pink: 2007 - preflare, black dashed: sum of VLBI flux).

the onset of the flare and one for October 2009 (see Figure 2, right panel). To this figure we have added the spectra of the VLBI components $\mathrm{C} 1$ and $\mathrm{C} 2$, with their flux densities determined from Gaussian model fits. The spectrum of the fainter southern VLBI component C2 is more inverted and peaks at a much higher frequency than $\mathrm{C} 1$, suggesting that $\mathrm{C} 2$ could be the VLBI core and not $\mathrm{C} 1$. In view of the orientation of the pc-scale jet, this however would require some extreme geometry. An alternative interpretation could be that $\mathrm{C} 2$ is a very compact and highly magnetized shock which is evolving. The continued mm-VLBI monitoring should clarify, if we see a moving or a stationary feature and if there is a relation with the gamma-ray activity observed at this date.

\section{Global VLBI at $1 \mathrm{~mm}$ wavelength}

The development of $1 \mathrm{~mm}$ VLBI started in the early 1990's and first transatlantic fringes were detected in 2002/2003 on a $8400 \mathrm{~km}$ long baseline between the IRAM $30 \mathrm{~m}$ telescope (Pico Veleta, 


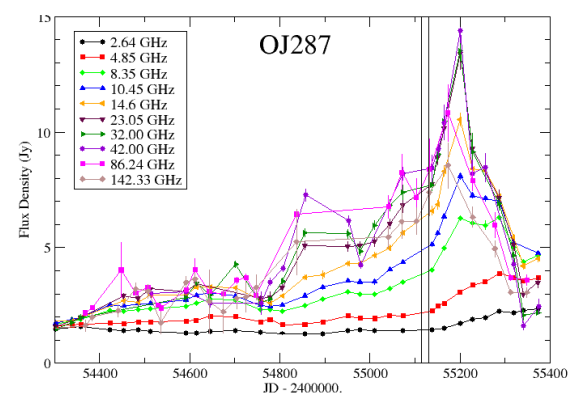

Figure 3: Radio variability of OJ287 at cm-wavelengths (data: F-GAMMA monitoring program [3]). A strong flux density peak is seen in early January 2010. The two black vertical lines bracket the times of the VLBI observations shown in Figure 1.

Spain) and the $10 \mathrm{~m}$ SMT (Mt. Graham, AZ, USA) ([6] and references therein). Recent progress in the development of digital VLBI recording systems has led to larger observing bandwidth (data rates) and higher sensitivities. The VLBI detection of ultra-compact emission regions in Sgr A* and M 87 at $230 \mathrm{GHz}$ and with telescopes in the USA (SMTO, SMA, CARMA) is another important step towards global mm- and sub-mm VLBI, which aims at the $\sim 10 \mu$ as scale imaging of the event horizon in nearby black holes ([4], Event Horizon Telescope: EHT).

In this context we begun in 2009 the planning, and in 2010 the outfit of the $12 \mathrm{~m}$ APEX telescope in Chile (Llano de Chajnantor, $5105 \mathrm{~m}$ ) for VLBI. In addition to the science case [7] our second motivation was the idea that APEX can serve as pathfinder for future mm-VLBI with ALMA. It took 2 years of work and one unsuccessful VLBI-test (in spring 2011), before we could claim the detection of first fringes with the APEX telescope. The setup and the observing details are presented in this conference by A. Roy et al. [8], and in [9]. On May 7, 2012 the bright quasar 3C $279\left(\mathrm{~S}_{230 \mathrm{GHz}}=19.8 \mathrm{Jy}\right)$ was observed with APEX and the SMTO (Arizona) in circular polarization and with the phased SMA (Hawaii) in linear polarization at a recording rate of $2 \mathrm{Gbps}$. On the baselines to APEX, fringes were detected with a typical SNR $\sim 10-15$ and on the SMTOSMA baseline the source was seen with SNR $\sim 30-35$. The detection of 3C 279 on the $7.22 \mathrm{G} \lambda$ long VLBI baseline between Chile and Hawaii forms a new world-record in angular resolution $(28.6 \mu$ as $)$ and demonstrates the feasibility of $1.3 \mathrm{~mm}$ VLBI even on the longest baselines. The amplitude calibration of the visibilities took into account all known effects and resulted in the visibilities shown in Fig. 4 (see [9] for details). In Fig. 5 we show a preliminary $1 \mathrm{~mm}$ VLBI map of 4 circular Gaussian components, which fit the visibilities and closure phases reasonably well ${ }^{1}$ $\left(\chi_{\text {reduced }}^{2}=2.42\right)$. Owing to the sparse uv-coverage and the residual calibration uncertainty, this model is not unambiguous and should be regarded with some care. A robust statement however seems to be, that 3C 279 appears elongated in North-South direction on the 0.1 mas scale. A similar elongation is also seen in a $3 \mathrm{~mm}$ VLBI image obtained with the GMVA only 10 days later (Fig. 6 , left panel). In the imaging process we were puzzled about a relatively low correlated flux at $230 \mathrm{GHz}$ of $\sim 1-2 \mathrm{Jy}$ at $\sim 3 \mathrm{G} \lambda$ and $\sim 0.7-1.2 \mathrm{Jy}$ at $\sim 7 \mathrm{G} \lambda$ (see Fig. 5, right panel). In Fig.6 (right panel) we therefore plot the brightness temperature for a few typical uv-distances. We also have added near-in time VLBI data at $86 \mathrm{GHz}$ (GMVA) and $43 \mathrm{GHz}$ (VLBA). It can be seen that

\footnotetext{
${ }^{1}$ Linear polarization at SMA may affect the closure phase if 3C 279 is highly polarized or antenna D-terms are large.
} 

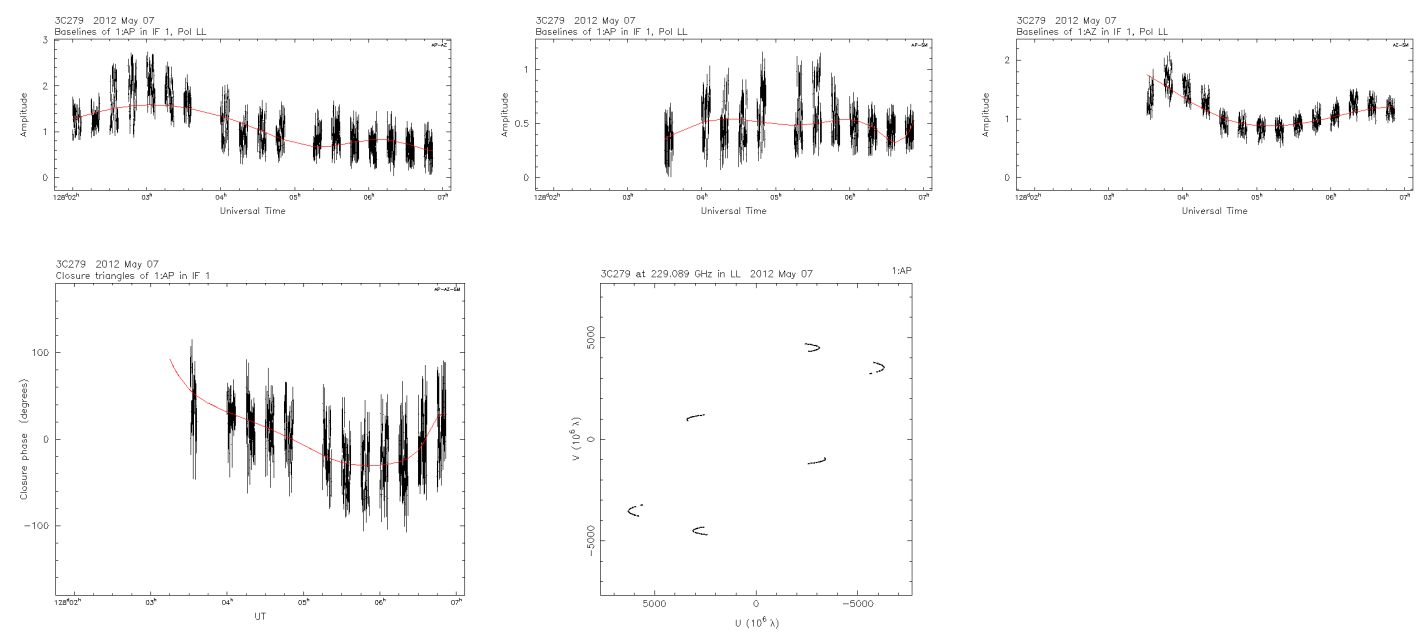

Figure 4: Top: Visibility amplitudes versus time at $230 \mathrm{GHz}$ and for the baselines APEX-SMTO (left), APEX-SMA (center), and SMTO-SMA (right). Bottom, left: Closure phase for this triangle. The red line shows the fit of the Gaussian model displayed in Fig. 5. Bottom, center: actual uv-coverage.
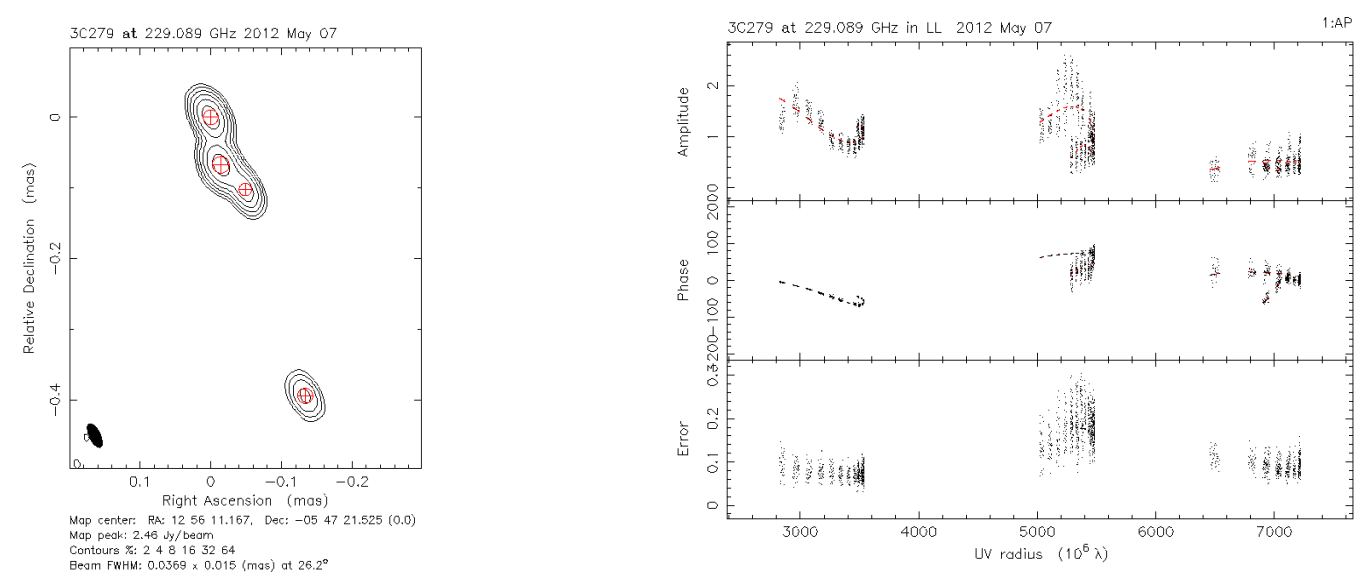

Figure 5: Left: Map of Gaussian modelfit components for $3 \mathrm{C} 279$ at $230 \mathrm{GHz}$. Four circular Gaussians, indicated by red circles with crosses, are fitted to the visibilities, see Fig. 4. The map is convolved with an observing beam (FWHM: 37 x $15 \mu$ as) from uniform weighting. Right: Visibility amplitude and phase plotted versus uv-distance. The red dashed lines show the fit of the Gaussian model to the data.

the brightness temperatures at $86 \mathrm{GHz}$ and $230 \mathrm{GHz}$ are very similar, but about a factor of $5-10$ lower than at $43 \mathrm{GHz}$. A jet brightness temperature which is decreasing with increasing frequency could indicate intrinsic jet acceleration or a decrease of the jet Doppler-factor towards the black hole.

\section{Future Outlook}

The obvious next steps for improving the capabilities of mm-VLBI are the addition of more sensitive telescopes and the further enhancement of array sensitivities. Since at mm-wavelengths weather is a limiting factor, triggered VLBI observations in pre-allocated longer time blocks are very feasible and require only moderate logistical efforts at the individual stations. The addition of 

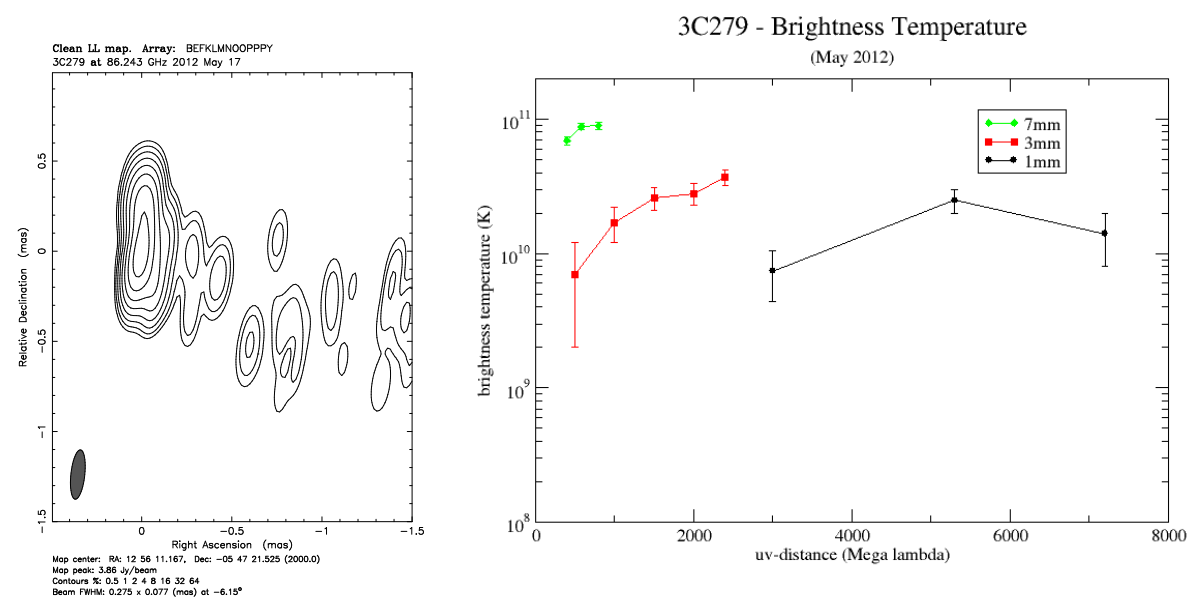

Figure 6: Left: 3mm VLBI map of 3C 279 observed with the GMVA on May 17, 2012. The size of the clean beam is $275 \times 77 \mu$ as (uniform weighting). Right: Brightness temperature of 3C 279 at $43 \mathrm{GHz}$ (green), $86 \mathrm{GHz}$ (red) and $229 \mathrm{GHz}$ (black) plotted versus projected baseline length (in $\mathrm{M} \lambda$ ). The data are all quasi-simultaneously obtained in early May 2012.

the phased ALMA, equivalent to a $\sim 85 \mathrm{~m}$ dish, to the existing VLBI arrays (at $v \geq 43 \mathrm{GHz}$ ) will lower the detection limits for mm-VLBI by up to two orders of magnitude (see [1] for the ALMA Phasing Project (APP)). Global mm-VLBI with ALMA however will become only possible, if in parallel to the ongoing technical development also a strong scientific user community not only obtains interesting results using the existing mm-VLBI arrays, but also actively pushes ALMA to this new frontier.

\section{References}

[1] W. Alef, et al., 2013, An ALMA beamformer for ultra high resolution VLBI and phased array science, in Proceedings of 11th EVN Symposium, POS (11th EVN Symposium) 053.

[2] I. Agudo, et al., 2011, Location of $\gamma$-ray Flare Emission in the Jet of the BL Lacertae Object OJ287 more than 14 pc from the Central Engine, ApJ 726, 13.

[3] L. Fuhrmann, et al., 2010, Recent multi-wavelength campaigns in the Fermi-GST era, arXiv:1007.0348 (2010).

[4] V. Fish, et al., 2013, New results from the Event Horizon Telescope, in Proceedings of 11th EVN Symposium, PoS(11th EVN Symposium) 056.

[5] J. Hodgson, et al., 2013, The latest results from the global mm VLBI array, in Proceedings of 11th EVN Symposium, POS (11th EVN Symposium) 096.

[6] T. P. Krichbaum, et al., 2008, Towards mm-VLBI, in proceedings of 9th EVN Symposium, PoS ( IX EVN Symposium) 001.

[7] T. P. Krichbaum, et al., 2010, Imaging Super Massive Black Holes and the Origin of Jets - Global mm- and sub-mm VLBI Studies of Compact Radio Sources, A Whitepaper and Proposal for submm-VLBI with APEX and ALMA, MPIfR, Bonn (download pdf).

[8] A. Roy, et al., 2013, VLBI at APEX: first fringes, in Proceedings of 11th EVN Symposium, PoS(11th EVN Symposium) 057.

[9] J. Wagner, et al., 2014, submitted to A\&A. 